\title{
Density functional theory study of Pt-induced Ge(001) reconstructions
}

\author{
Danny E. P. Vanpoucke and Geert Brocks \\ Computational Materials Science, Faculty of Science and Technology and MESA + Institute for Nanotechnology, University of Twente, \\ P.O. Box 217, 7500 AE Enschede, The Netherlands
}

(Received 15 September 2009; published 26 January 2010)

\begin{abstract}
Pt deposited on a Ge(001) surface spontaneously forms nanowire arrays. These nanowires are thermodynamically stable and can be hundreds of atoms long. The nanowires only occur on a reconstructed Pt-Gesurface where they fill the troughs between the dimer rows on the surface. This unique connection between the nanowires and the underlying substrate make a thorough understanding of the latter necessary for understanding the growth of the nanowires. In this paper we study possible surface reconstructions containing 0.25 and 0.5 of a monolayer of Pt. Comparison of calculated scanning tunneling microscope (STM) images to experimental STM images of the surface reconstruction reveal that the Pt atoms are located in the top layer, creating a structure with rows of alternating Pt-Ge and Ge-Ge dimers in a $c(4 \times 2)$ arrangement. Our results also show that Pt atoms in the second or third layer cannot be responsible for the experimentally observed STM images.
\end{abstract}

DOI: 10.1103/PhysRevB.81.035333

PACS number(s): 61.72.uf, 68.35.B-, 68.37.Ef

\section{INTRODUCTION}

Metal/semiconductor interface interactions continue to attract interest in both fundamental and applied research and are of vital importance for the semiconductor and microelectronics industry. With miniaturization pushing into the nanoscale regime, new ways of constructing nanoscale devices are investigated. Bottom-up approaches such as atomic size reconstructions, self assembly and metal nanowires (NWs) are investigated as alternatives for the usual top-down techniques such as etching and lithography. ${ }^{1}$ Generally, the focus lies on metal/Si interfaces because of the importance of $\mathrm{Si}$ in the semiconductor industry. Of these, the $\mathrm{Au} / \mathrm{Si}(001)$ interface, due to the high conductivity of $\mathrm{Au}$, is one of the most well studied interfaces.

For this system, it has been shown that different types of reconstructions appear depending on the Au coverage and the annealing temperature. ${ }^{2-4}$ The metal/Ge interface is less well studied, notwithstanding its importance in the development of radiation detector systems and high speed electronic devices. Recent experimental studies of the $\mathrm{Pt} / \mathrm{Ge}(001)$ interface, done at the submonolayer $\mathrm{Pt}$ coverage regime, have shown the formation of self-organized Pt NW arrays after high temperature annealing. ${ }^{5-7}$ It has been suggested that this reconstruction was due to a strengthened interaction of the $5 d$ metals with the Ge surface: relativistic mass effects which contract the $s$ shell, reducing its energy and thus increase the $s$ occupancy at the expense of the $d$ electrons. This partially depletes the antibonding $d$ states, strengthening the $d$ bonds. ${ }^{8}$ The same mechanism was thought to be responsible for the one dimensional chain structures of $\mathrm{Au}$ on Ge. ${ }^{9,10}$ There certainly are similarities between the growth of $\mathrm{Au}$ and $\mathrm{Pt}$ chains on $\mathrm{Ge}(001)$, such as the appearance of dimer vacancies before the chains appear. Yet, there are also important differences between the two systems. The Pt NWs only form after annealing at over $1000 \mathrm{~K}$, while the Au chains appear after deposition at $675 \mathrm{~K}$ and disappear into the bulk Ge when annealed at $1000 \mathrm{~K} .{ }^{5,10}$ Moreover, while the Pt NWs have a thickness of a single atom, the Au chains are dimer rows containing $\mathrm{Au}-\mathrm{Au}$ or $\mathrm{Au}-\mathrm{Ge}$ dimers. ${ }^{9}$ The Pt NWs are thermodynamically stable and their length is only limited by the size of the underlying terrace. This so-called $\beta$ terrace is a previously unknown Pt-Ge-surface reconstruction of which, to date, only a tentative model proposed by Gürlü $e t$ $a l .{ }^{5}$ exists. The unique connection between this terrace and the NWs makes a thorough understanding of the reconstruction desirable before one can study and understand the NW arrays and the physical phenomena associated with them. ${ }^{11,12}$

In this work, we present a geometry for the $\beta$ terrace based on the comparison of the simulated scanning tunneling microscope (STM) images (henceforth referred to as pseudoSTM images) to experimental STM images. The current experimental information on the $\beta$ terraces is very limited. At the moment of writing only STM images are available. A preliminary account of this work appeared in Ref. 11.

The structure of this paper is as follows: in Sec. II we describe the theoretical method and present the notation used for the different geometries. In Sec. III we present our theoretical results starting with a comparison of the formation energies of the different geometries followed by a general review of the pseudo-STM images. We identify one particular geometry as the structure of the $\beta$ terrace, which is discussed in more detail. As a last point of Sec. III, we take a look at some geometries containing $\mathrm{Pt}$ atoms in the second and third layer and show that these cannot be responsible for the $\beta$-terrace STM pictures, but they could be responsible for the indented dimer and two dimer vacancy images seen in the STM pictures of a different $\mathrm{Pt}$ induced $\mathrm{Ge}(001)$ reconstruction, the so-called $\alpha$ terrace. ${ }^{5}$ Finally, in Sec. IV the conclusions are given.

\section{THEORETICAL METHOD}

\section{A. Setup}

Density functional theory (DFT) calculations are carried out using the projector augmented waves (PAWs) method and the local density approximation (LDA) functional with the exchange and correlation function calculated by Ceperley and Alder (CA), as implemented in the VASP program. ${ }^{13-17} \mathrm{~A}$ 


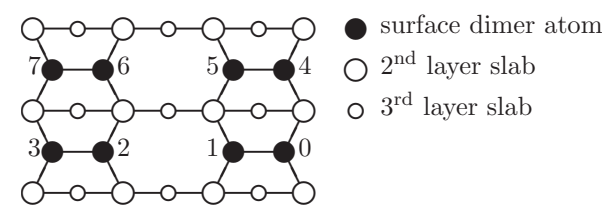

FIG. 1. Top view of the Ge(001) surface with symmetric (2 $\times 1)$ reconstruction. 0-7 are indexes for the surface dimer atoms and are used in the nomenclature of the geometries (see text).

plane-wave basis set with kinetic-energy cutoff of $287 \mathrm{eV}$ is applied and the semicore $d$ shell of Ge is included in the valence shell for better accuracy. The surface is modeled by periodically repeated slabs of 12 layers of Ge atoms. Extensive convergence tests, showed no advantage in time/ accuracy for a H-passivated $\mathrm{Ge}$ slab over a symmetric $\mathrm{Ge}$ slab. Based on the advantages of the higher symmetry of the symmetric slab, we choose to use the later. The slabs are then mirrored in the $z$ direction with reconstructions on both surfaces, in which specific Ge atoms are replaced by $\mathrm{Pt}$ atoms. A vacuum region of $\sim 15.5 \AA$ is used to separate the slabs along the $z$ axis. The Brillouin zone $(B Z)$ of the $(2 \times 4)$ surface unit cell is sampled using a $6 \times 3$ Monkhorst-Pack special $k$-point mesh. ${ }^{18}$ To optimize the geometry of the slabs the conjugate gradient method is used. The positions of the $\mathrm{Ge}$ atoms in the two center layers are fixed as to represent the bulk phase of the system.

\section{B. $\mathrm{Ge}(001)$-surface and nomenclature}

To model the $\beta$ terrace we first model the clean $\mathrm{Ge}(001)$ surface. Both the buckled $b(2 \times 1)$ and $c(4 \times 2)$ reconstructions using a $(4 \times 2)$ supercell and the symmetric $(2 \times 1)$ reconstruction were generated using a $(2 \times 1)$ supercell. After relaxation, the $b(2 \times 1)$ and the $c(4 \times 2)$ reconstructions were found to be 0.302 and $0.374 \mathrm{eV}$ per dimer lower in energy than the symmetric $(2 \times 1)$ dimer reconstruction. A buckling angle of $\sim 19.5^{\circ}$ was found for both asymmetric reconstructions, and a dimer length of $2.45 \AA$ for the $b(2$ $\times 1)$ reconstruction and of $2.51 \AA$ for the $c(4 \times 2)$ reconstruction was calculated, all in accordance with previous theoretical and experimental results. ${ }^{19-22}$

Based on experimental evidence, ${ }^{5,6}$ we assume that the $\beta$ terrace contains 0.25 monolayer (ML) of Pt. Furthermore, we assume that these $\mathrm{Pt}$ atoms are located in the top layer of the Ge slab. We will show in Sec. III D that Pt in the second or third layer cannot be responsible for the experimentally obtained STM images.

To model the $\beta$ terrace, all possible nonisomorphic geometries are examined. We generate these geometries by substitution of two surface Ge atoms, of the $b(2 \times 1)$ surface reconstruction in the $(4 \times 2)$ supercell, by $\mathrm{Pt}$ atoms. Ignoring the buckling of the Ge dimers for a moment, this leads to seven nonisomorphic geometries. The first $\mathrm{Pt}$ atom replaces the Ge surface atom in position 0 , while the second $\mathrm{Pt}$ atom replaces one of the other seven surface Ge atoms (cf. Fig. 1). We will refer to these geometries as $\beta_{1}$ to $\beta_{7}$. If the buckling of the Ge dimers in the $b(2 \times 1)$ reconstruction is taken into account, some of the symmetry is lost. Let us assume for a moment that the atoms of the dimers in Fig. 1 with an even index are the down dimer atoms and those with an odd index are the up dimer atoms. In what follows we will keep referring to those positions in this manner. We now notice that in some geometries both $\mathrm{Pt}$ atoms are located at an up (down)dimer atom position and in other cases one Pt atom is located in an up dimer atom position while the other $\mathrm{Pt}$ atom is located in a down dimer atom position. ${ }^{23}$ While for the latter all combinations that are isomorphic stay isomorphic when tilting is taken into account this is not the case in the former situation. Take for example the $\beta_{2}$ geometry with its Pt atoms in positions 0 and 2. An isomorphic geometry in the symmetric reconstruction is one with $\mathrm{Pt}$ atoms in positions 1 and 3 . In the $b(2 \times 1)$ geometry, as described above, the first $\beta_{2}$ geometry has both $\mathrm{Pt}$ atoms at down dimer atom sites, while the second has them at up dimer atom sites. To distinguish these two different geometries, indices $u$ and $d$ are used to indicate the up or down location of the Pt atoms. So the first $\beta_{2}$ geometry is called $\beta_{2 d}$ and the second one $\beta_{2 u}{ }^{24}$ For geometries with one atom at an up and one at a down dimer atom site both the $u$ and $d$ index are used to indicate just this (e.g., $\left.\beta_{3} \rightarrow \beta_{3 u d}\right)$. This results into ten nonisomorphic geometries to start from.

To complete the picture of this system we also included possible geometries containing 0.5 ML of Pt in the top layer. Such a surface then consists entirely of Pt-Ge dimers. Geometries containing pure Pt and pure Ge dimers were not considered based on our results for the $\beta$ geometries.

The three possible geometries considered are a $2 \times 1$ reconstruction $\left(\gamma_{1}\right)$ with all Pt atoms at the even (or odd) positions, a $2 \times 2$ reconstruction $\left(\gamma_{2}\right)$ with the Pt atoms at positions $0,2,5$, and 7 , and a $4 \times 2$ reconstruction $\left(\gamma_{3}\right)$ with the $\mathrm{Pt}$ atoms at positions $0,3,5$, and 6. All these geometries were optimized using the conjugate gradient method while keeping the positions of the Ge atoms in the two center layers fixed.

\section{THEORETICAL RESULTS}

\section{A. Geometry and formation energy}

\section{Stability of the $\beta$ geometries}

The stability of the different geometries was investigated by comparison of their formation energies to the formation energy of the Ge $b(2 \times 1)$ surface reconstruction. The formation energy of a certain geometry, $\beta_{\mathrm{x}}$, can be calculated using the expression: ${ }^{25}$

$$
E_{f}=\left[\left(E_{\beta_{\mathrm{x}}}+N_{\mathrm{Pt}} E_{\mathrm{Ge}}\right)-\left(E_{b(2 \times 1)}+N_{\mathrm{Pt}} E_{\mathrm{Pt}}\right)\right] / N_{\text {Pt-dimers }},
$$

with $E_{\beta_{\mathrm{x}}}$ the total energy of the $\beta_{\mathrm{x}}$ geometry, $N_{\mathrm{Pt}}$ the number of $\mathrm{Pt}$ atoms in the system, $E_{\mathrm{Ge}}$ and $E_{\mathrm{Pt}}$ the bulk energy of a $\mathrm{Ge}$ and a $\mathrm{Pt}$ atom, $E_{b(2 \times 1)}$ the total energy of the Ge $b(2$ $\times 1)$ surface reconstruction, and $N_{\mathrm{Pt} \text {-dimers }}$ the number of surface dimers containing $\mathrm{Pt}$ atoms. Negative values for $E_{f}$ indicate an increase in stability caused by replacing a Ge atom by a Pt atom.

Table I clearly shows that formation energy of the $\beta_{1 u d}$-surface is much higher than that of the other structures. This is the only structure containing a Pt-Pt dimer; all the 
TABLE I. The formation energy and dimer buckling angles of the $\beta$ and $\gamma$ surfaces compared to $\mathrm{Ge}(001)$ surface reconstructions. For the $\beta_{1 u d}$ geometry the Pt-Ge dimer buckling refers to the buckling of the Pt-Pt dimer and the value between brackets for the Ge-Ge buckling refers to the buckling of the Ge-Ge dimers located in the same dimer row as the Pt-Pt dimers. The notations $(u)$ and $(d)$ in the column of the Pt-Ge buckling refer to the position of the Pt atom in the dimer, while in the Ge-Ge buckling column they indicate if the Ge dimer is in a dimer row with the Pt atom in the up or down position (except for $\beta_{5 u d}$ where it refers to the location of the $\mathrm{Pt}$ atom in a Pt-Ge dimer in the adjacent dimer row). For $\gamma_{2}$ and $\gamma_{3}$ the buckling angles of both dimers of a single dimer row are given.

\begin{tabular}{|c|c|c|c|}
\hline & $\begin{array}{c}E_{f} \\
(\mathrm{meV})\end{array}$ & $\begin{array}{l}\text { Pt-Ge buckling } \\
\text { (degrees) }\end{array}$ & $\begin{array}{c}\text { Ge-Ge buckling } \\
\text { (degrees) }\end{array}$ \\
\hline$b(2 \times 1)$ & 0 & & 19.42 \\
\hline$c(4 \times 2)$ & -73 & & 19.67 \\
\hline$\beta_{1 u d}$ & 589 & 20.57 & $19.07(18.25)$ \\
\hline$\beta_{2 u}$ & -16 & -4.42 & 21.03 \\
\hline$\beta_{2 d}$ & 94 & -0.15 & 18.23 \\
\hline$\beta_{3 u d}$ & 34 & $\begin{array}{l}-4.43(u) \\
-1.82(d)\end{array}$ & $\begin{array}{l}21.85(u) \\
18.13(d)\end{array}$ \\
\hline$\beta_{4 u}$ & -61 & 1.92 & 18.85 \\
\hline$\beta_{4 d}$ & -25 & 0.50 & 18.85 \\
\hline$\beta_{5 u d}$ & 83 & $\begin{array}{r}-5.04(u) \\
7.38(d)\end{array}$ & $\begin{array}{l}19.06(u) \\
18.43(d)\end{array}$ \\
\hline$\beta_{6 u}$ & -25 & -4.25 & 20.82 \\
\hline$\beta_{6 d}$ & 82 & -0.88 & 18.13 \\
\hline$\beta_{7 u d}$ & 29 & $\begin{array}{l}-4.83(u) \\
-1.30(d)\end{array}$ & $\begin{array}{l}21.43(u) \\
18.06(d)\end{array}$ \\
\hline$\gamma_{1}(2 \times 1)$ & -47 & 1.95 & \\
\hline$\gamma_{2}(2 \times 2)$ & 80 & $6.13,-6.46$ & \\
\hline$\gamma_{3}(4 \times 2)$ & 89 & $5.75,-6.11$ & \\
\hline
\end{tabular}

other surface models contain mixed Pt-Ge dimers. It shows that replacing a $\mathrm{Ge}$ atom in a Pt-Ge dimer with a Pt atom will require about 0.5 to $0.6 \mathrm{eV}$. This allows us to rule out the formation of Pt-Pt dimers in the $\beta$ terrace. Table I also shows a preference for geometries which contain Pt-Ge dimer rows with the Pt atom in the up position. The entire set of formation energies, with the exception of $\beta_{1 u d}$, is spread over a relatively small energy interval of $\sim 150 \mathrm{meV}$, which is of the same order of magnitude as the thermal energy corresponding to the annealing temperature. In the experiment, the germanium samples on which the $\mathrm{Pt}$ is deposited need to be annealed at a temperature of $>1000 \mathrm{~K}(\approx 86 \mathrm{meV})$ before the $\beta$ terrace (and the nanowires) appear. ${ }^{5}$ This makes it impossible to exclude any structures based solely on energetics with the exception of the $\beta_{1 u d}$ structure.

All formation energies are given per Pt containing dimer. This means that the formation energy is the average energy to form a Pt-Ge dimer in the $\beta_{2}-\beta_{7}$ structures. ${ }^{25}$ In the structures indicated with the $u d$ subscript the Pt-Ge dimers are not equivalent. To make a distinction between these two types of dimers we will refer to a Pt-Ge dimer with the Pt atom in the up position as a Pt-up dimer, and similar for a Pt-down dimer. The $\beta_{3 u d}$ structure contains a Pt-up and a Pt-down dimer, while the $\beta_{2 u}$ and $\beta_{2 d}$ structures contain either only Pt-up or Pt-down dimers.

If we take the average of the formation energy of a Pt-up and a Pt-down dimer from the $\beta_{2 u}$ and $\beta_{2 d}$ structures we get $39 \mathrm{meV}$, comparable to the formation energy of an average Pt-Ge dimer in the $\beta_{3 u d}$ structure. Comparison of the $\beta_{7 u d}$ structure with the $\beta_{6 u}$ and $\beta_{6 d}$ structures gives a similar result, $28.5 \mathrm{meV}$ for the average of the $\beta_{6 u}$ and $\beta_{6 d}$ structure compared to $29 \mathrm{meV}$ for the average $\beta_{7 u d} \mathrm{Pt}-\mathrm{Ge}$ dimer. The pattern of Pt-Ge and Ge-Ge dimers in these two sets of three geometries is the same, indicating that the energy due to interactions between neighboring dimer rows is small compared to the formation energy. In contrast, the formation energy of the Pt-Ge dimer depends strongly on the surrounding dimers within the dimer row.

The third triplet of similarly patterned structures $\left(\beta_{5 u d}\right.$, $\beta_{4 u}$, and $\beta_{4 d}$ ) shows this last point even more clearly. In this case the average of the formation energy of a Pt-Ge dimer of the $\beta_{4 u}$ and $\beta_{4 d}$ structures is $-43 \mathrm{meV}$, much less than the average formation energy for a Pt-Ge dimer in the $\beta_{5 u d}$ structure. Unlike the previous examples, the Pt-Ge dimers of the $\beta_{5 u d}$ structure have a different surrounding along the dimer row than those in the $\beta_{4}$ structures: antiparallel instead of parallel Pt-Ge dimers. This shows that the interaction between neighboring dimers in a single dimer row is strong, and provides a large contribution to the formation energy of a Pt-Ge dimer. It also shows that rotation of a Pt-Ge dimer in the $\beta_{5 u d}$-surface cell would give an energy gain of roughly $0.2-0.3 \mathrm{eV}$ per surface unit cell.

Based on such comparisons we can make an estimate of the inter and intra dimer row energy contributions to the formation energy of a Pt-Ge dimer, $E_{f}$. To estimate the interaction energy between neighboring dimer rows, we compare structures with different interdimer row neighbors for the Pt-Ge dimers. Comparison of the $\beta_{2}$ to the $\beta_{6}$ structures, the $\beta_{3 u d}$ to the $\beta_{7 u d}$ structure, the $\beta_{4}$ to the $\gamma_{1}$ structure, the $\gamma_{2}$ to the $\gamma_{3}$ structure, and $\beta_{5 u d}$ to the $\gamma_{2}$ and $\gamma_{3}$ structures, shows that this interaction energy is of the order of about $5-10 \mathrm{meV}$ per Pt-Ge dimer.

A similar estimate can be made for the interaction energy along a dimer row by comparing structures with different neighbors in a single dimer row for a Pt-Ge dimer. Comparing the $\beta_{4}$ to the $\beta_{6}$ and the $\beta_{5 u d}$ structures, and comparing the $\gamma_{1}$ to the $\gamma_{2}$ and $\gamma_{3}$ structures, gives an interaction energy in the range of $50-150 \mathrm{meV}$ per Pt-Ge dimer, about one order of magnitude larger than the interdimer row interaction energy. It also shows that the proximity of the second $\mathrm{Pt}$ atom has a large influence, both for better $\left(\beta_{4 u}\right)$ and for worse $\left(\beta_{5 u d}\right)$, on the formation energy. In case of the $\beta_{4 u}$ reconstruction all $\mathrm{Pt}$ atoms are located at the same side of the dimer row while for the $\beta_{5 u d}$ reconstruction the Ge and $\mathrm{Pt}$ atoms of the dimer rows have a checkerboard pattern with the resulting surface strain deteriorating the formation energy.

Furthermore, we found that the length of the dimers was not influenced by the different surface structures, with the sole exception of $\beta_{1 u d}$, which is the only surface structure containing a Pt-Pt dimer. The lengths of the Ge-Ge dimers were found to be $2.45 \pm 0.01 \AA\left(2.34 \pm 0.03 \AA\right.$ for $\left.\beta_{1 u d}\right)$ and 
for the Pt-Ge dimers $2.35 \AA(2.58 \AA$ for the Pt-Pt dimer in $\left.\beta_{1 u d}\right)$. The buckling angles on the other hand do show a dependence on the location of nearby Pt atoms. Noteworthy is the fact that the Pt-Ge dimers show little to no buckling and the little buckling effect they show depends on the location of the Pt atom: if the Pt atom is located in the up position then the buckling angle is about $4-5^{\circ}$, while it is less than $2^{\circ}$ if the $\mathrm{Pt}$ atom is located in the down position. The sign used for the buckling angles indicates wether dimers are buckled in the same (same sign) or opposite (opposite sign) direction of the Ge surface dimers. In all cases, except $\beta_{1 u d}$ and $\beta_{4}$, the Pt-Ge dimers are buckled in the opposite direction of the Ge-Ge dimers thus creating a $(2 \times 2)$ or $(4 \times 2)$ surface reconstruction. These reconstructions also modify the Ge positions in the second and third layer in a similar way as found for the Ge $c(4 \times 2)$ surface reconstruction, which will be discussed in more detail in Sec. III C. ${ }^{19,22}$ Table I also shows that the Ge-Ge dimers are slightly influenced by the Pt-Ge dimers in the same dimer row, increasing the buckling angle to about $21-22^{\circ}$ for Pt-Ge dimers with the Pt atom in the up position and decreasing it to about $18^{\circ}$ if the $\mathrm{Pt}$ atom in the Pt-Ge dimer is in the down position.

Another difference between Pt-Ge dimers with the Pt atom in the up or down position becomes clear when the geometry of the dimers of a dimer row containing both Pt-Ge and Ge-Ge dimers is investigated. The Pt-Ge dimer shifts orthogonal to the dimer row. If the Pt atom is located in the up position this shift is very small $(\sim 0.03 \AA)$ and almost entirely due to the $\mathrm{Pt}$ atom moving in the direction of the up position. On the other hand, if the $\mathrm{Pt}$ atom is located in the down position, the shift is in the down direction and both the $\mathrm{Ge}$ and the Pt atom of the Pt-Ge dimer are shifted over a considerably larger distance of $\sim 0.4 \AA$.

Comparison of the average $z$ position of the dimers shows the Pt-Ge dimers to be 0.01 to $0.14 \AA$ lower than the $\mathrm{Ge}-\mathrm{Ge}$ dimers for each of the considered structures. All the geometrical information of the Pt-Ge dimers combined with the formation energies given in Table I shows a preference for Pt atoms to be surrounded by Ge atoms which stick further out of the surface than these Pt atoms. This agrees with the preference for Ge-terminated surfaces in Pt/Ge alloys. ${ }^{26}$

\section{Stability of the $\gamma$ geometries}

The stability of the $\gamma$ geometries is investigated the same way as for the $\beta$ geometries. Equation (1) is used to calculate the formation energy per Pt containing surface dimer, making close comparison with the $\beta$ geometries possible. ${ }^{25}$ In the previous paragraph we found the inter dimer row interaction to be very small, compared to the intradimer row interaction. This allows us to compare the $\gamma_{1}$ geometry to the $\beta_{4 \mathrm{x}}$ geometries, and the $\gamma_{2}$ and $\gamma_{3}$ geometries to the $\beta_{5 u d}$ geometry. The main difference between these geometries is the presence of a Ge-Ge dimer row in the $\beta$ geometries. Table I shows the formation energies for the $\gamma$ geometries to be in the range expected from the comparable $\beta$ geometries. As was shown in the previous paragraph, the interaction between neighboring dimer rows is relatively small for $\beta_{5 u d}$, $\gamma_{2}$, and $\gamma_{3}$ geometries, and a larger part of the formation energy can be attributed to strain between sequential Pt-Ge dimers. For the $\beta_{4 \mathrm{x}}$ and $\gamma_{1}$ geometries the influence of the neighboring dimer rows is more important. Although the geometry of the Pt-Ge dimer in the $\gamma_{1}$ and $\beta_{4 u}$ geometry is nearly indistinguishable, and thus quite different from that of the one in the $\beta_{4 d}$ geometry, the formation energy is about the average of that of the $\beta_{4 u}$ and the $\beta_{4 d}$ geometry. The geometries of the Pt-Ge dimers in the $\beta_{5 u d}, \gamma_{2}$, and $\gamma_{3}$ geometries are almost identical and the angle between the antiphase Pt-Ge dimers in a dimer row is in each case about $12^{\circ}$ with the Pt atoms on the geometrically lower side of the dimer. Furthermore, looking at the positions of sequential dimers in a dimer row, a shift orthogonal to the dimer row is observed. This shift increases the distance between the $\mathrm{Pt}$ atoms with $0.56-0.61 \AA$ in the direction orthogonal to the dimer row. It is larger than any of such shifts produced with normal Ge-Ge dimers in the dimer row, indicating the crucial role of the Pt atoms.

\section{B. Electronic structure}

\section{General properties of the $\beta$ geometries}

Since there is no clear evidence based on energetics alone to put forward a single reconstruction as the $\beta$ terrace, a more direct comparison with the STM observations is needed. This is done by calculating the local density of states (LDOS) $\rho(\mathbf{r}, \varepsilon){ }^{27}$ The theoretical (integrated) LDOS is calculated as $\bar{\rho}(\mathbf{r}, \varepsilon) \propto \int_{\varepsilon}^{\varepsilon_{F}} \rho\left(\mathbf{r}, \varepsilon^{\prime}\right) d \varepsilon^{\prime}$, with $\varepsilon_{F}$ the Fermi energy. A surface of constant density is then constructed according to $\bar{\rho}(x, y, z, \varepsilon)=C$, with $C$ a real constant. This construction gives the height $z$, as a function of the lateral position $(x, y)$, which is mapped linearly onto a gray scale. This mimics the presentation of STM images in the usual constant-current mode of operation.

Figure 2 shows pseudo-STM images of the $\beta_{2 u}, \beta_{2 d}$, and $\beta_{7 u d}$ surfaces for both filled and empty states. The filled-state images of the $\beta_{2 u}$ surface show a bright peak above the up atom of the Ge dimer and a slightly less bright peak above the Ge atom of the Pt-Ge dimer. This creates a zigzag pattern of bright spots (of two different intensities). Comparison of the filled-state pictures of the $\beta_{2 u}$ and $\beta_{2 d}$ surfaces, shows images for the Pt-Ge dimers that are clearly different. Where there is a clear peak when the Pt atom was located between up-Ge atoms $\left(\beta_{2 u}\right)$ there is a relatively evenly spread rectangular feature when the $\mathrm{Pt}$ atom is located between down-Ge atoms $\left(\beta_{2 d}\right)$. This feature is spread out over both atoms of the $\mathrm{Pt}-\mathrm{Ge}$ dimer and is slightly higher at the position of the $\mathrm{Ge}$ atom. The difference in height of the feature at the $(x, y)$ positions of the dimer atoms is five times smaller than the difference in height between similar points of the feature produced by the Ge dimer. The Pt-Ge feature, in the $\beta_{2 d}$ filled-state picture, has a height equal to the average height of the Ge dimer and does not protrude as far in the trough between the dimer rows as the Ge dimer. This gives the overall impression of "battlements" [cf. all dimer rows in Fig. 2(c) and the upper and lower dimer row in Fig. 2(e)].

The $\beta_{7 u d}$ surface contains both a Pt atom between up-Ge atoms and one between down-Ge atoms. As could be expected from the filled-state images of the $\beta_{2 u}$ and $\beta_{2 d}$ surfaces the filled-state image of the $\beta_{7 u d}$ surface shows a zig- 


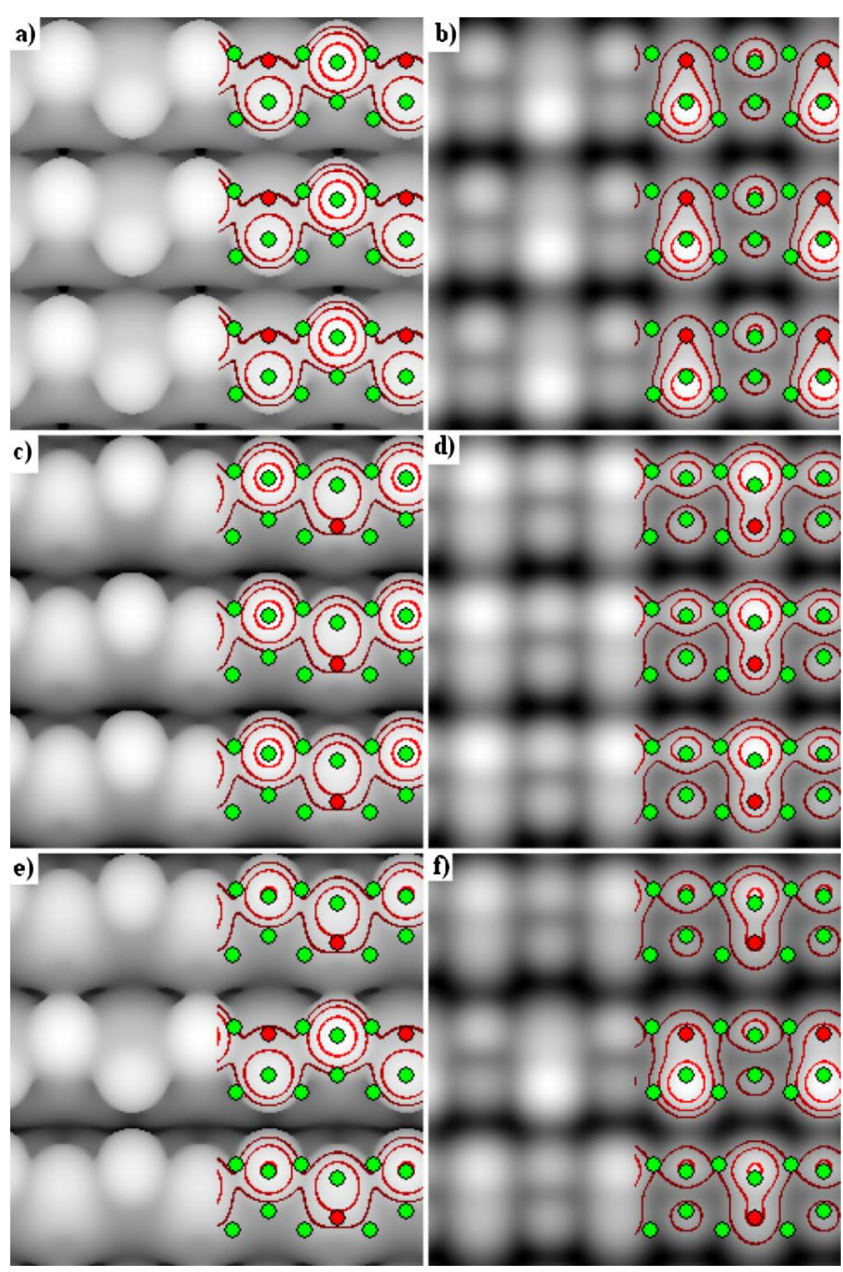

FIG. 2. (Color online) Pseudo-STM images showing filled (a), (c), (e) and empty (b), (d), (f) states of the $\beta_{2 u}$ (a), (b), $\beta_{2 d}$ (c), (d), and $\beta_{7 u d}$ (e), (f) geometries. For the filled-state images $\varepsilon=\varepsilon_{F}$ $-0.70 \mathrm{eV}$ and for the empty-state images $\varepsilon=\varepsilon_{F}+1.50 \mathrm{eV}$ is used. The constant density is chosen such that the maximum $z$ is at $3.0 \AA$ above the highest atom. The small dark gray (red) filled circles represent the Pt atoms in the top layer, the small light gray (green) circles represent the positions of the Ge atoms in the two top layers. Contours are added to indicate the main features discussed in the text.

zag row and a battlement row alternatingly. A further study of the pseudo-STM images of the other $\beta$ geometries shows these same structures reappearing in different combinations.

A Pt atom located between up-Ge atoms causes a feature which is about three times as steep as the feature caused by a $\mathrm{Pt}$ atom between down-Ge atoms. The Pt atom is also always located at the lower end of these features, even though sometimes the geometry shows the Pt atom to be the higher atom of the dimer. For the $\beta_{4 \mathrm{x}}$ geometries, the dimer row of the Pt-Ge dimers shows a $b(2 \times 1)$ structure while a $(2 \times 2)$ structure is found in case of the $\beta_{5 u d}$ geometry. This Pt-Ge dimer row is always much dimmer than the Ge dimer row, but it still shows nicely distinct dimer patterns. Another careful look at the pseudo-STM structure of a Pt-Ge dimer with the Pt atom between up-Ge atoms shows that unlike the Gedimer this structure has a dim tail directed toward the $\mathrm{Pt}$ atom. Although this is best visible in the empty-state pictures

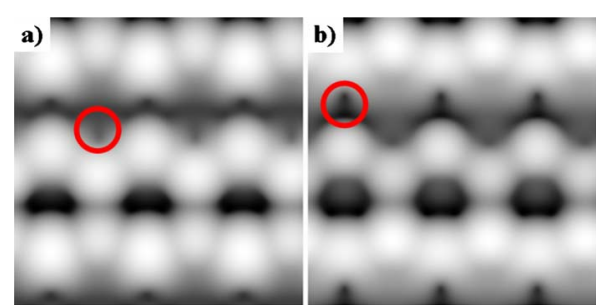

FIG. 3. (Color online) Pseudo-STM images showing (a) filled and (b) empty states of the $\beta_{7 u d}$ geometry close to the Fermi level, with $\varepsilon=\varepsilon_{F} \pm 0.30 \mathrm{eV}$. The maximum $z$ is at $4 \AA$ above the highest atom. There is a clear difference between the dimer rows with the $\mathrm{Pt}$ atoms located between up-Ge atoms (middle zigzag row) and the dimer rows with the Pt atoms located between down-Ge atoms (top and bottom row). A notch (circle) in the constant density surface above the location of the Pt atoms is visible for both the filled- and empty-state image.

of Fig. 2, it is present for both filled- and empty-state images.

The above analysis of the pseudo-STM images shows that the filled-state images of single dimers can be used as building blocks to reconstruct the geometry of the $\beta$ terrace (this will be shown in Sec. III B 3).

The empty-state pseudo-STM images for a bias far above the Fermi level show a very simple picture, with little or no dependence of the position and size of the Pt atom features on the neighboring Ge atoms. The Ge dimer shows up as a set of two separate peaks, while the Pt-Ge dimer is smeared out, showing a bright ball on the side of the Ge atom and a dim wide tail on the side of the Pt atom. Though the Pt-Ge dimer images look very similar in the empty-state pseudoSTM pictures, they are not exactly the same. The empty-state picture of $\beta_{7 u d}$ in Fig. 2 shows clearly that the Pt-Ge dimer with $\mathrm{Pt}$ in the up position is slightly brighter and has a somewhat more egg-like shape. Close to the Fermi level, the pseudo-STM images of filled and empty states are nearly indistinguishable and some interesting features appear in the pseudo-STM images. The most eye-catching feature is the notch in the isosurface, indicated by a circle in Figs. 3(a) and 3(b). It is caused by the Pt atom at that location. Though clearest for the Pt atom between up-Ge atoms in a filled-state images and clearest for the Pt atom between down-Ge atoms in the empty-state images, both are present at either side of the Fermi level and were found in all geometries. Checking contour plots of cross-sections in the $z$ direction at the position of these notches, showed they are caused by the gap between two lobes of the $d$ orbitals of the Pt atom underneath.

\section{General properties of the $\gamma$ geometries}

The pseudo-STM pictures of the $\gamma$ geometries show in general the same image for the Pt-Ge dimer rows as was seen for the corresponding Pt-Ge dimer rows in the $\beta_{4 \mathrm{x}}$ and $\beta_{5 u d}$ pictures (cf. Fig. 4). For the $\gamma_{1}$ geometry, the pseudo-STM pictures show dimer rows with a bright spot at the Ge atom position. This spot becomes sharper when the bias becomes smaller, both above and below the Fermi energy. The $\gamma_{2}$ and $\gamma_{3}$ geometries show nearly identical pictures [cf. Figs. 4(d) and $4(\mathrm{e})]$, the main difference being the phaseshift between 


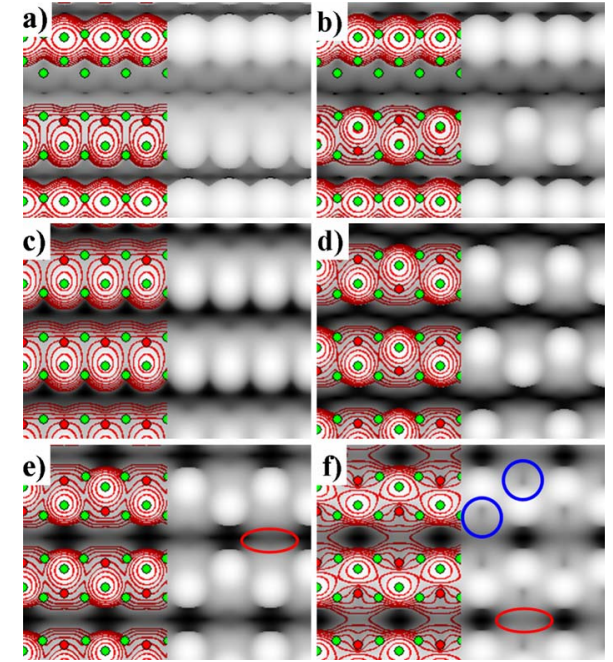

FIG. 4. (Color online) Comparison of $\beta$ and $\gamma$ structures. Filled state pseudo-STM images of the $\beta_{4 u}$ (a), $\beta_{5 u d}(\mathrm{~b}), \gamma_{1}(\mathrm{c}), \gamma_{2}$ (d), and $\gamma_{3}$ structure (e) with $\varepsilon=\varepsilon_{F}-0.70 \mathrm{eV}$ and an empty-state pseudoSTM image of the $\gamma_{3}$ structure (f) close to the Fermi level with $\varepsilon$ $=\varepsilon_{F}+0.30 \mathrm{eV}$. The maximum $z$ is at $3 \AA$ above the highest atom. The green/red (light/dark gray) disks indicate the positions of the $\mathrm{Ge}(\mathrm{Pt})$ atoms in the two top layers of the system. Contours separated $0.2 \AA$ are added to guide the eye. The circles indicate the "notch," while the ellipse shows the position of the "bridge." The Ge dimer row in (a) and (b) show slightly broader and higher dimer images than the Pt-Ge dimer row.

neighboring dimer rows, which causes the appearance of a very dim (at least $1.5 \AA$ lower than the dimer peaks in the pseudo-STM pictures) bridge between the Pt atoms of neighboring dimer rows, indicated with an ellipse in Figs. 4(e) and 4(f). This bridge is most clear in the empty-state pictures. Again it is the Ge side of the Pt-Ge dimers which lights up very bright, causing a zigzag pattern of bright spots along each dimer row. For small biases this zigzag pattern becomes more pronounced and also the notch above the $\mathrm{Pt}$ atoms appears, as indicated with the circles in Fig. 4(f). At a large negative bias the bright spot has a dim tail directed toward the Pt atom. All Pt-Ge dimer images for $\gamma_{2}$ and $\gamma_{3}$ are identical, unlike for the $\beta$ geometries.

\section{3. $\beta_{6 u}$ structure as geometry for the $\beta$ terrace}

In this paragraph we will show that, although most $\beta$ structures studied are energetically available, only the $\beta_{6 u}$ geometry can be related to the experimentally observed $\beta$ terrace. Figures 5 and 6 show (experimental) STM images of the $\beta$ terrace at biases of -0.30 and $+0.30 \mathrm{~V}$. The filled-state image clearly shows two types of dimers alternating in each dimer row. One of the dimer types shows up as a big bright spot on one side of the dimer row while the other dimer type shows up as a smaller somewhat dimmer spot on the other side of the dimer row, with a dim tail extending to the opposite side of the dimer row. These are the only two types of dimers appearing in the image. Comparing this to the results of the pseudo-STM images seen in the previous paragraph, the first type of dimer is the Ge-Ge dimer while the second type of dimer presents all the typical features of the Pt-Ge

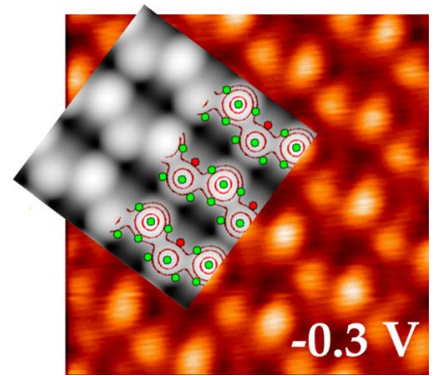

FIG. 5. (Color online) Comparison of the filled-state pseudoSTM image of the $\beta_{6 u}$ geometry (gray scale overlay) with the corresponding experimental STM image of the $\beta$ terrace. The STM image is obtained using a sample bias of $-0.3 \mathrm{~V}$, while the pseudoSTM image is generated using $\varepsilon=\varepsilon_{F}-0.70 \mathrm{eV}$ and a maximum $z$ of $4 \AA$ above the highest atom. The small red (dark gray) disks represent the positions of the $\mathrm{Pt}$ atoms in the top layer, while the green (light gray) disks represent the positions of the $\mathrm{Ge}$ atoms in the top two layers. The QDRs are clearly visible in both STM images, as is the presence of two different types of dimer.

dimer with the Pt atom located between up-Ge atoms. This allows us to exclude all $\beta$ geometries containing Pt at a down location, leaving only the $\beta_{2 u}, \beta_{4 u}$, and $\beta_{6 u}$ geometries. Taking the relative ordering of the features of neighboring dimer rows into account, the geometry which represents the $\beta$ terrace can only be the $\beta_{6 u}$ geometry. Filled- and empty-state pseudo-STM images of the $\beta_{6 u}$ geometry are displayed on top of the experimental pictures in Figs. 5 and 6.The emptystate pseudo-STM image (Fig. 6) shows some very specific features of this geometry. There are triangular holes where the Pt atom is located and the Ge atoms of the top layer also create a triangular feature dominated by the zigzag structure of the up-Ge atoms and the Ge atoms in the Pt-Ge dimers. Comparison of the empty-state images in Fig. 6 reveals the existence of these features in both images, further strengthening our belief that the $\beta_{6 u}$ geometry is the geometry of the $\beta$ terrace. Another look at Table I also confirms this geometry to be more stable than the Ge $b(2 \times 1)$ surface reconstruction. Furthermore, for the $\beta_{6 \mathrm{x}}$ geometries, the Pt atoms are also distributed in the most homogeneous way. The pseudo-STM images show only for the $\beta_{6 u}$ geometry this level of agreement with the experimental STM images, al-

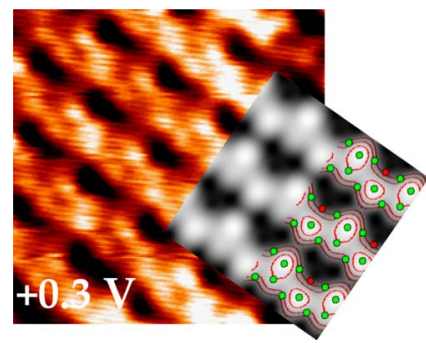

FIG. 6. (Color online) Same as Fig. 5 but now for the emptystate images. The sample bias used for the STM image is $+0.3 \mathrm{~V}$ and $\varepsilon=\varepsilon_{F}+0.70 \mathrm{eV}$ is used for the pseudo-STM image. Note the triangular holes above the Pt atoms (red disks) which are present in both the theoretical and the experimental STM image. This is a unique feature for the $\beta_{6 u}$ structure. 


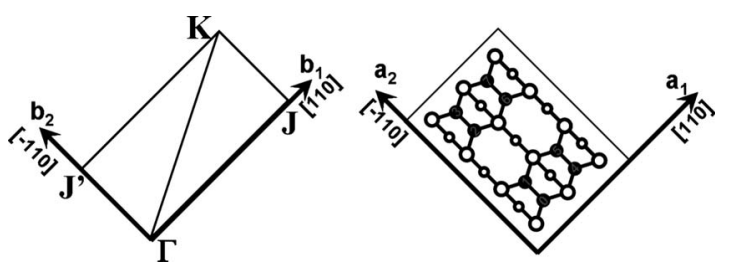

FIG. 7. High symmetry lines and points of the surface Brillouin zone (left) for the $\beta$ structures. A surface unit cell is shown on the right, indicating the orientation of the dimer rows.

lowing the $\beta_{6 u}$ structure to be presented as the geometry of the experimentally observed $\beta$ terrace.

Figure 8(b) shows the band structure of the $\beta_{6 u}$ geometry along the high-symmetry lines $\Gamma-J-K-J^{\prime}-\Gamma-K$ of the surface BZ (cf. Fig. 7). Figure 8(a) shows the band structure of the $\mathrm{Ge}(001) c(4 \times 2)$ reconstruction along the same lines for comparison. In general, the two band structures show similar behavior. Perpendicular to the dimer row direction, along the $J-K$ and $J^{\prime}-\Gamma$ line, the bands show little dispersion. Along the $J^{\prime}-\Gamma$ line, a few of these bands cross the Fermi level. The main difference between the two band structures is located along the $\Gamma-J$ and $K-J^{\prime}$ lines. For the $\beta_{6 u}$ structure we find bands with a strong dispersion opposite to what is seen for the $\mathrm{Ge}(001)$ surface. These bands are indicated with the ellipses in Fig. 8(b). Furthermore, these bands also cross the
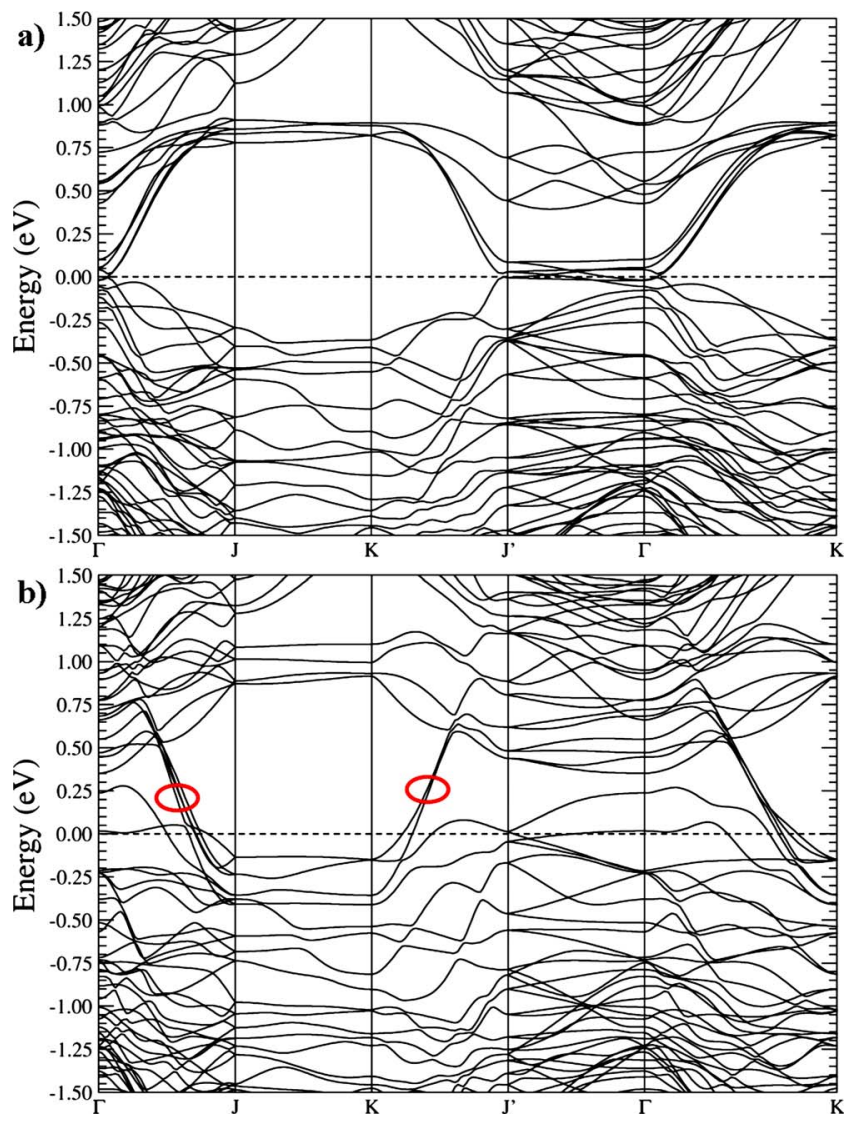

FIG. 8. (Color online) Comparison of the band structure, along high-symmetry lines (cf. Fig. 7), of the $\mathrm{Ge}(001) c(4 \times 2)$ reconstruction (a) and the $\beta_{6 u}$ geometry (b). The energy zero is set at the Fermi level.
Fermi level in the second half of the $\Gamma-J$ line and in the first half of the $K-J^{\prime}$ line, resulting in the metallic behavior observed for the $\beta$ terrace. ${ }^{28}$ Taking a closer look at these specific bands near the crossing of the Fermi level, we find that their character contains three main components. The largest component has a Pt-like character. The other two (smaller) components have a Ge-like character: more specifically, the second layer $\mathrm{Ge}$ atoms bound to the $\mathrm{Pt}$ atoms in the top layer and the Ge-up atom of the Ge surface dimer that is located in between the Pt atoms. The orbital character of the Pt contribution is mainly $p_{x}, p_{y}$ and $d_{x z}, d_{y z}$, making it highly planar. The Ge contribution depends on the $\mathrm{Ge}$ atoms involved. The orbital character of the contribution of second layer Ge atoms is mainly $s p^{3}$, while the top layer Ge atoms bound to the $\mathrm{Pt}$ atom contribute mainly a $p_{z}$ orbital character, indicating the presence of a $\pi$ bond with energy close to the Fermi level for the Pt-Ge dimer. Interestingly, the Ge-up atoms of the Ge dimers in between Pt atoms along the dimer row have a significant contribution to most of the bands we are interested in. The orbital character in this case is mostly $p_{x}$ and $p_{y}$. The presence of bands close to (and crossing) the Fermi level connected to an orbital character directed along the dimer row direction, indicates the possible presence of surface conduction channels along this direction. These bands could be the source of the confined states observed in Refs. 6 and 29 .

Figure 9 shows the density of states (DOS) of the $\beta_{6 u}$ geometry and the LDOS of the surface atoms. ${ }^{30}$ The total DOS roughly follows the DOS observed for bulk Ge, as could be expected. The main difference is found in the band gap (BG) region. Here, three distinct peaks can be observed, indicated by the labels 1, 2, and 3 in Fig. 9. The two outer peaks (1 and 3) are also observed for a reconstructed $\mathrm{Ge}(001)$ surface, where they are linked to the dangling bonds of the Ge surface dimer atoms. Since half the surface dimers of the $\beta_{6 u}$ geometry are Ge dimers, it is not surprising to observe these states. Figure 9(b) shows the LDOS of the up and down atoms of the Ge surface dimers. Comparison to the $\mathrm{Ge}(001) c(4 \times 2)$ and $b(2 \times 1)$ reconstruction, shows the peak due to the Ge down atom (3) to be quite broad, with a width comparable to what is found for the $b(2 \times 1)$ reconstruction. This broadening probably originates from the small tilt angle of the Pt-Ge dimers. This makes the angle between subsequent dimers in a quasidimer row (QDR) smaller, making it more comparable to the zero angle of the $b(2 \times 1)$ reconstruction. The Ge up atom shows two peaks in the BG (1 and 2 in Fig. 9(b)). The strong peak, labeled 1 is also found for the Ge up atom of the $\mathrm{Ge}(001) c(4 \times 2)$ and $b(2$ $\times 1)$ reconstructions. The weaker second peak has a nonzero value for the DOS at the Fermi level and is one of the contributions to peak 2 in the total DOS.

The LDOS image of the Ge atoms bound directly to the $\mathrm{Pt}$ atom shows something interesting [cf. Fig. 9(c)]. The Ge atom of the Pt-Ge dimer also has two peaks in the BG region, contributing to peaks 2 and 3 in the total DOS. A sharp peak around the same value as seen for the Ge down atom at the conduction band (CB) side (3) and a second peak near the valence band (VB) side of the BG region (2). This second peak is located just at the Fermi level. Between these two peaks the LDOS goes to zero, just above the Fermi level. 


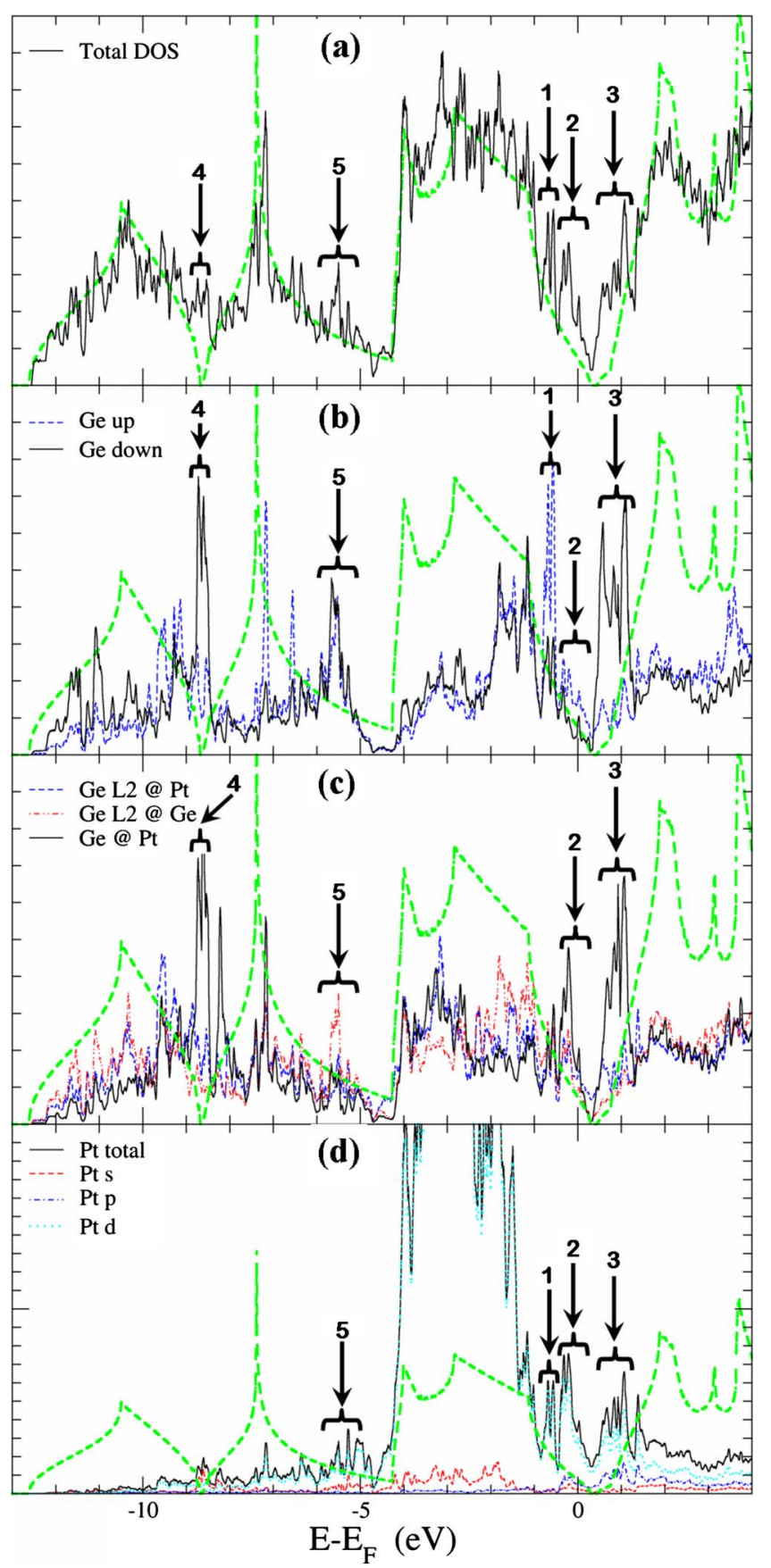

FIG. 9. (Color online) Total DOS of the $\beta_{6 u}$ geometry and the LDOS of the surface atoms. The bold dashed green curve shows the DOS of bulk Ge, it is shifted to align the edges of the BG regions. The labeled peaks are discussed in the text.

The second layer Ge atoms (indicated with L 2 in Fig. 9) show roughly the same structure, both have a small contribution to the conducting peak 2 of the total DOS. However, in the $\mathrm{BG}$ region it are only the second layer Ge atoms bound to the $\mathrm{Pt}$ atoms of the top layer that have nonzero values over the entire BG region [cf. (blue) dashed curve in Fig. 9(c)]. Second layer Ge atoms bound to the Ge atoms of the top layer have a LDOS which goes to zero. The LDOS for the Pt atoms, presented in Fig. 9(d), shows the presence of $\mathrm{Pt} d$ states at and around the Fermi level, with three peaks at the same locations as found for the Ge atoms bound to these $\mathrm{Pt}$ atoms. For the Ge atoms, it are mainly $p$ states contributing to these peaks near the Fermi level, showing clearly the presence of a hybridization of Ge $p$ with Pt $d$ states. Together with the Ge-up atoms and $\mathrm{Pt}$ atoms, the $\mathrm{Ge}$ atoms bound to the Pt atoms form a corridor of "metallic" atoms along the edge of the QDR, giving rise to the metallic peak 2 of the total DOS, in agreement with the experimental observations. ${ }^{28}$ The additional peaks, 4 and 5 , in the total DOS are related to the surface Ge atoms, and are also observed for the $\mathrm{Ge}(001) c(4 \times 2)$ and $b(2 \times 1)$ reconstructions.

\section{Experimental existence of other $\beta$ geometries}

Although the comparison between experimental and pseudo-STM images in Figs. 5 and 6 shows clearly that the clean $\beta$ terrace has a $\beta_{6 u}$ geometry, the formation energies given in Table I indicate that also other geometries should be observed experimentally. In particular the $\beta_{4 u}$ structure has a slightly lower energy. The latter structure leads to STM images that are completely different to what is observed experimentally, as do other non- $\beta_{6 u}$ structures. Our calculations therefore indicate that the formation of such structures is kinetically hindered. Investigation of experimental STM images has shown the existence of (small) regions where dimer rows contain a mix of different (non- $\beta_{6 u}$ ) geometries. These geometries were mostly found near defects in the normal $\beta$ terrace, such as dimer vacancies and the end points of a NW. Because these geometries seem related to defects, we will not go into the details of their geometry and focus solely on the $\beta_{6 u}$ geometry.

\section{C. $\boldsymbol{\beta}_{6 u}$ geometry}

To characterize the geometry of the $\beta_{6 u}$ structure, it is compared to the geometry of the $\mathrm{Ge}(001) b(2 \times 1)$ and $c(4$ $\times 2$ ) reconstructions. This shows a slight shift of the second and third layer $\mathrm{Ge}$ atoms of the $\beta_{6 u}$ structure in the surface plane with regard to the $\mathrm{Ge} b(2 \times 1)$ equilibrium positions. In what follows we will show that this deformation of the geometry can be understood as a combination of three geometric modifications, each linked to a symmetry breaking of the top layer structure.

In the second layer of the $\beta_{6 u}$ geometry, two inequivalent sites are present: (i) a Ge atom between a top layer Ge up atom and a Pt atom and (ii) a Ge atom between a top layer Ge down atom and a Ge atom of a Pt-Ge dimer. The Ge atom at the latter site is displaced toward the neighboring $\mathrm{Ge}$ atom of the Pt-Ge dimer, while the Ge atom at the former site is displaced toward the neighboring Ge up atom (cf. Fig. 10). In comparison to the Ge $b(2 \times 1)$ reconstruction, in the surface plane these atoms appear as twisted around the in-plane position of the third layer Ge atom in between them. For this reason, such a modification of the geometry, i.e., the twisting of the tetrahedral structure, is known as twisting. 22

The Ge $(2 \times 2)$ surface reconstruction, for which a diagrammatic representation can be found in Fig. 2 of Ref. 22, is a simple Ge surface reconstruction which only shows the twisting modification with regard to the $b(2 \times 1)$ reconstruction. Twisting is believed to be a strain release mechanism, 


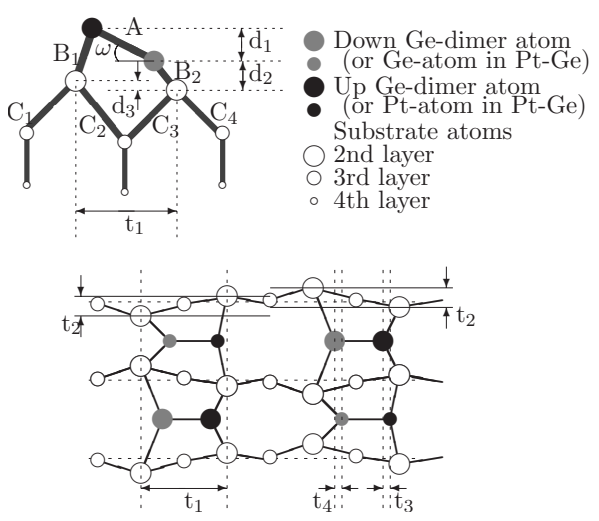

FIG. 10. Top: diagram showing a side view of a relaxed Ge-Ge dimer, including the labeling used for both Ge-Ge and Ge-Pt surface dimers. Bottom: top view schematic representation of the $\beta_{6 u}$ reconstruction.

where strain (orthogonal to the surface) due to different surface dimer buckling is (partially) compensated by an inplane modification of the second layer geometry, indicated with $t_{2}$ in Fig. 10. This means that the twisting parameter $t_{2}$ is related to the relative buckling angle of sequential surface dimers in a dimer row. Assuming a linear relation as first order approximation one finds $t_{2} \sim\left|\omega_{\operatorname{dimer} 1}-\omega_{\operatorname{dimer} 2}\right|=\Delta \omega$, where $\omega_{\text {dimer } X}$ is the buckling angle $\omega$ shown in Table II for each of the dimers involved. Because the relative angle between sequential dimers can be used as a measure for the twisting, it becomes possible to estimate the twisting for similar surfaces via the ratio of their relative buckling angle

$$
R=\frac{t_{2}^{\mathrm{rec} 1}}{t_{2}^{\mathrm{rec} 2}}=\frac{\Delta \omega^{\mathrm{rec} 1}}{\Delta \omega^{\mathrm{rec} 2}},
$$

where rec 1 and rec 2 refer to each of the two reconstructions being compared.

Unlike the twisting observed for Ge surface reconstructions with differently buckled surface dimers, the twisting found in the $\beta_{6 u}$ geometry is asymmetric. From Fig. 10 follows that $t_{2}$ can be considered to consist of two parts; the component along the dimer row direction of the bond $\mathrm{C}_{2}$ and the component along the dimer row direction of the bond $\mathrm{C}_{3}$. The ratio of the contributions of $\mathrm{C}_{2}$ and $\mathrm{C}_{3}$ to $t_{2}$ is $1: 1$ for the $\mathrm{Ge}(2 \times 2)$ and $\mathrm{Ge} c(4 \times 2)$ reconstructions, while it is 6.3:9 for the $\beta_{6 u}$ reconstruction. This means that, in comparison to the $\mathrm{Ge} c(4 \times 2)$ reconstruction, the second layer $\mathrm{Ge}$ atom between the Pt atom and the Ge up atom is pulled slightly back toward the Pt atom in the surface plane.

Using Eq. (2) to calculate the ratio $R$ of the twisting of the $\beta_{6 u}$ reconstruction to the $\mathrm{Ge} c(4 \times 2)$ reconstruction making use of the values for $t_{2}$ presented in Table II we find a value of 0.5406 for $R$. However, making use of the relative buckling angle we find a value of 0.6395 for $R$. Unlike the $\mathrm{Ge}$ $c(4 \times 2)$ reconstruction, there are different kinds of surface dimers on the $\beta_{6 u}$ surface. To include this inhomogeneity of the surface dimers in Eq. (2), we add weight factors to the angle contributions. We found the mass percentages of the dimers to be good weight factors. This leads to a twisting ratio for the $\beta_{6 u}$ to the $\mathrm{Ge} c(4 \times 2)$ structure:

$$
\begin{aligned}
R & =\frac{\left|\left(\frac{M_{\mathrm{Ge}}+M_{\mathrm{Pt}}}{3 M_{\mathrm{Ge}}+M_{\mathrm{Pt}}}\right) \omega_{\beta_{6 u} \mathrm{Pt}}-\left(\frac{2 M_{\mathrm{Ge}}}{3 M_{\mathrm{Ge}}+M_{\mathrm{Pt}}}\right) \omega_{\beta_{6 u} \mathrm{Ge}}\right|}{\omega_{c(4 \times 2)}} \\
& =\frac{t_{2}^{\beta_{6 u}}}{t_{2}^{\operatorname{Gec}(4 \times 2)}},
\end{aligned}
$$

with $M_{\mathrm{x}}$ the atomic mass of a single (average) atom of type $x$, and $\omega_{\mathrm{y}}$ the angle $\omega$ shown in Table II for the specific dimer $y$. Using Eq. (3) a value of 0.5142 is found for the ratio R, in agreement with the value obtained for the ratio of the $t_{2}$ parameters. Also for other $\beta$ structures, equations equivalent to Eq. (3) give an improvement of the estimated ratios of $t_{2}$ over the ratios obtained through Eq. (2). For example, for the $\beta_{1 u d}$ structure compared to the Ge $c(4 \times 2)$ structure, the ratio of the twisting parameters $t_{2}$ is 0.5477 . Using the relative buckling angles in Eq. (2) one finds a value of 0.0354, which is much too small. However, using the weighted buckling angles, as in Eq. (3), one finds a value of 0.5121 , in good agreement with the ratio of the parameters $t_{2}$.

This shows $t_{2}$ to be a useful parameter for comparison of the asymmetry induced strain in these systems. As a result, the difference of the twisting parameter, $t_{2}$, between the $\mathrm{Ge}$ $c(4 \times 2)$ and $\beta_{6 u}$ reconstruction can be attributed to the introduced $\mathrm{Pt}$ atoms. On the one hand by leveling out the $\mathrm{Pt}-\mathrm{Ge}$ dimer, i.e., the $\omega$ term and on the other hand through the weight factor.

In conclusion, twisting is the result of the relative buckling angle of sequential dimers in a dimer row which is influenced strongly by the Pt atoms present in the $\beta_{6 u}$ structure.

Figure 11 gives diagrams of the top view of a row of second and third layer Ge atoms in geometries with increasing complexity. The horizontal dashed lines give an arbitrary zero-line with regard to which all amplitudes are given. The $\mathrm{Ge}(2 \times 2)$-reconstruction shows the simple image of twisting of the tetrahedral arrangement, discussed above. The image of the $\mathrm{Ge} c(4 \times 2)$ reconstruction presents, in addition to

TABLE II. Bond lengths and distances in $\AA$ and the dimer tilt angle $\omega$ in degrees. All as defined in Fig. 10.

\begin{tabular}{lccccccccccccccc}
\hline \hline & $\omega$ & $\mathrm{A}$ & $\mathrm{B}_{1}$ & $\mathrm{~B}_{2}$ & $\mathrm{C}_{1}$ & $\mathrm{C}_{2}$ & $\mathrm{C}_{3}$ & $\mathrm{C}_{4}$ & $d_{1}$ & $d_{2}$ & $d_{3}$ & $t_{1}$ & $t_{2}$ & $t_{3}$ & $t_{4}$ \\
\hline Ge $b(2 \times 1)$ & 19.42 & 2.450 & 2.512 & 2.426 & 2.475 & 2.449 & 2.543 & 2.475 & 0.815 & 0.775 & 0.099 & 3.818 & 0 & 0 & 0 \\
Ge $c(4 \times 2)$ & 19.60 & 2.511 & 2.506 & 2.430 & 2.463 & 2.481 & 2.481 & 2.462 & 0.842 & 0.749 & 0.007 & 3.817 & 0.283 & 0.335 & 0.332 \\
$\beta_{6 u}$ Ge dimer & 20.82 & 2.462 & 2.552 & 2.433 & 2.479 & 2.435 & 2.492 & 2.469 & 0.875 & 0.731 & 0.027 & 3.764 & 0.153 & 0.049 & 0.003 \\
$\beta_{6 u}$ Pt dimer & -4.25 & 2.354 & 2.378 & 2.521 & 2.479 & 2.435 & 2.492 & 2.469 & 0.174 & 1.358 & 0.027 & 3.764 & 0.153 & 0.049 & 0.003 \\
\hline \hline
\end{tabular}




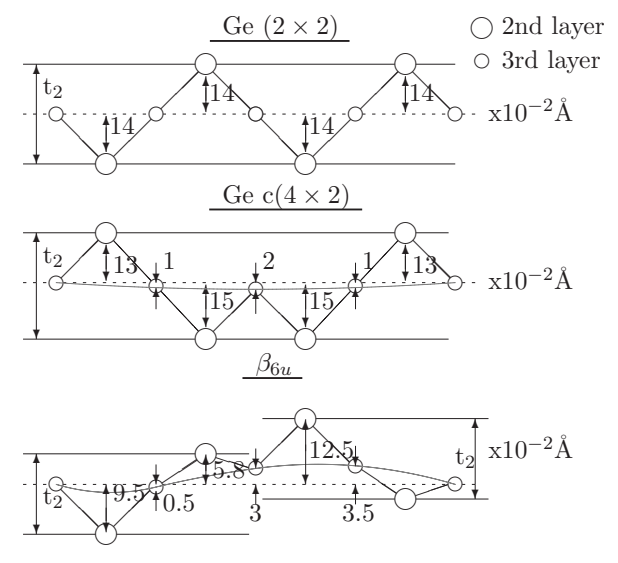

FIG. 11. The diagrams show a top view of the upper row, as represented in the diagram of the $\beta_{6 u}$ reconstruction in Fig. 10, of second and third layer $\mathrm{Ge}$ atoms in a $\mathrm{Ge}(2 \times 2)$, $\mathrm{Ge} c(4 \times 2)$, and $\beta_{6 u}$ reconstruction. The adjacent top layer atoms, as represented in the bottom diagram of Fig. 10, are then from left to right: Ge (2 $\times 2$ ): Ge up-dimer atom, down-dimer atom, up-dimer atom and down-dimer atom. Ge $c(4 \times 2)$ : Ge down-dimer atom, up-dimer atom, up-dimer atom, and down-dimer atom. $\beta_{6 u}$ : Ge atom, Pt atom, Ge down-dimer atom, and Ge up-dimer atom. [Data for the Ge $(2 \times 2)$ reconstruction is taken from Gay et al. (Ref. 22)].

twisting, a bending of the entire second and third layer structure, indicated with the gray curve in Fig. 11. It shows a slight displacement of the third layer Ge atoms in the plane along the dimer row direction. This is due to the asymmetry between the $\mathrm{C}_{1}, \mathrm{C}_{4}$ and the $\mathrm{C}_{2}, \mathrm{C}_{3}$ bonds as a result of the opposite buckling of dimers in adjacent dimer rows.

In the $\beta_{6 u}$ structure, just like the Ge $c(4 \times 2)$ reconstruction, the surface dimers in adjacent dimer rows show an opposite buckling which results in a bending, as shown with the gray curve in the bottom picture of Fig. 11. Furthermore, the presence of $\mathrm{Pt}$ atoms in the top layer dimers seems to increase the amplitude of the bending by a factor of two and the extrema of the bending curve move from the third layer atoms between the dimer rows to the third layer atoms underneath the dimer rows.

The presence of two different dimer types in the $\beta_{6 u}$ structure complicates this image even further, as can be seen in the bottom picture of Fig. 11. On top of the previous two distortions a shift of the twisting $\mathrm{t}_{2}$ between adjacent dimer rows is present. Shift is the displacement of the horizontal lines defining the twisting parameter $t_{2}$ between neighboring dimer rows (cf. bottom pictures of Figs. 10 and 11), in other words; it is the shift along the dimer row direction of the twisting parameter $t_{2}$ its bounding box between adjacent dimer rows. This shift is a consequence of the induced asymmetry in the components along the dimer row direction of the $\mathrm{C}_{2}$ and $\mathrm{C}_{3}$ bonds contributing to $t_{2}$, which in turn is due to the asymmetry of having different dimer types in a dimer row, as well as in adjacent dimer rows.

In case of the $\beta_{6 u}$ structure, the shift is directed toward the Pt-Ge dimer, away from the Ge-Ge dimer (cf. Fig. 10, bottom picture). This makes shift, which is due to different dimer types in one system, the third surface modification present in the $\beta_{6 u}$ geometry. It shrinks the surface cell, formed by the first and second layer atoms, containing the

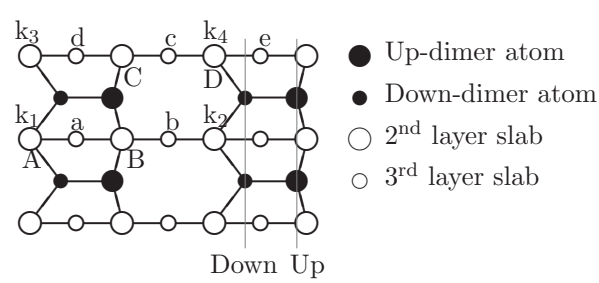

FIG. 12. Locations of Ge atoms substituted by Pt atoms in the second (capital) and third (small) layer. The second layer Ge atoms pushed to the surface by third layer Pt atoms are marked by the $k_{\mathrm{x}}$.

Pt-Ge dimer in favor of the Ge-Ge dimer surface cell. Table II also shows a decrease in the parameter $t_{1}$, reducing the size of the Pt-Ge dimer surface cell even further and partially compensating the expansion of the Ge-Ge dimer surface cell.

The bond length of the Ge dimer in the $\beta_{6 u}$ reconstruction is only slightly longer than it was in the $b(2 \times 1)$ reconstruction but still significantly shorter than it is in the $c(4 \times 2)$ reconstruction. The accompanying change in buckling angle accounts for this slight elongation. Also, the back bonds of the Ge atoms of all dimers are longer than their counterparts in the asymmetric Ge surface reconstructions. This while the height of these back bonds for the Ge-Ge dimers is similar to the one found for the $c(4 \times 2)$-reconstruction. $t_{3}$ and $t_{4}$, shown in Fig. 10, give the in-plane displacement along the surface dimer bond direction between sequential dimers in a single dimer row, i.e., the alignment of the surface atoms along the dimer rows. Although they are not zero, they are at least one order of magnitude smaller than the values obtained for the $c(4 \times 2)$ reconstruction, and unlike the $c(4 \times 2)$ reconstruction there is an asymmetry between the two displacements. This results from the difference in length of Pt-Ge and Ge-Ge bonds.

The $\mathrm{C}_{n}$ bonds between the second and third layer Ge atoms have lost all symmetry, this is due to the two types of surface dimers present in the $\beta_{6 u}$ structure. This is unlike the reconstructions studied by Gay et $a .^{22}$ where only one type of surface dimer is present in each system and as a consequence the $\mathrm{C}_{n}$ bonds show much symmetry.

\section{Other geometries}

Though the previous section shows clearly that the $\beta$ terrace has a $\beta_{6 u}$ geometry, it is still interesting to have a look at some geometries containing $\mathrm{Pt}$ atoms in the second or third layer of the system. Previous calculations and experimental observations have shown that Pt atoms can also be found in subsurface positions. ${ }^{31}$ Before the transformation to the $\beta$ terrace, there is what Gürlü et al. call an $\alpha$ terrace. This type of terrace is characterized by a large number of dimer vacancy defects. Also, in this initial stage of $\mathrm{Pt}$ growth on $\mathrm{Ge}(001)$ there are still a number of features which are not entirely understood, such as "indented dimers."

Figure 12 shows the positions where Ge atoms were substituted with $\mathrm{Pt}$ atoms in the second and third layer of the system. The final geometries were obtained the same way as the $\beta$ geometries, i.e., substitution of $\mathrm{Ge}$ atoms by $\mathrm{Pt}$ atoms in a $\mathrm{Ge}(001) b(2 \times 1)$ reconstructed slab and relaxation of the 
TABLE III. Formation energy per Pt atom of reconstructions containing $\mathrm{Pt}$ atoms in the second and third layer. The Pt positions are shown in Fig. 12. The asterisk indicates that the Ge atoms at the $k_{\mathrm{x}}$ positions (between brackets) are removed from the system, and the values were obtained after relaxation of the modified system.

\begin{tabular}{ccr}
\hline \hline Layer & Pt position(s) & $\begin{array}{c}E_{f} \\
(\mathrm{meV})\end{array}$ \\
\hline 2 & A (down) & 24 \\
2 & B (up) & -40 \\
2 & A \& D (down) & -4 \\
2 & B \& C (up) & -163 \\
3 & a (row) & -1115 \\
$3^{*}\left(k_{1}\right)$ & a (row) & -155 \\
3 & b (trough) & 68 \\
3 & a and b & -630 \\
$3^{*}\left(k_{1} \& k_{2}\right)$ & a and b & 467 \\
3 & a and c & -467 \\
$3^{*}\left(k_{1}\right)$ & a and c & -66 \\
3 & a and d & -616 \\
$3^{*}\left(k_{1} \& k_{3}\right)$ & a and d & -32 \\
3 & a and e & -1141 \\
$3^{*}\left(k_{1} \& k_{4}\right)$ & a and e & -346 \\
\hline \hline
\end{tabular}

geometry using the conjugate gradient method keeping the positions of the two center layers fixed.

\section{Pt in the second layer}

When Ge atoms in the second layer are substituted with Pt atoms, there are two possible inequivalent sites to consider. One under the down-Ge atoms and one under the up-Ge atoms of the surface dimers. Table III shows the formation energy per Pt atom for these two locations differs only 64 $\mathrm{meV}$ and $\mathrm{Pt}$ favors the position under the up-Ge dimer atoms. Table III also shows that the introduction of a second $\mathrm{Pt}$ atom in the second layer improves the stability of the system considerably, increasing the formation energy by 28 and 123 $\mathrm{meV}$ per Pt atom. This difference from what would be expected from the single Pt substitution results shows there is a positive correlation between the Pt atoms in the second layer. Furthermore, comparison of Tables I and III shows the formation energies per surface unit are roughly in the same range as the formation energies per surface unit of the studied $\beta$ and $\gamma$ geometries, making these structures energetically also viable candidates for the $\beta$ terraces. Pseudo-STM images on the other hand clearly show this is not the case.

The top part of Fig. 13 shows the influence of the substitution of a single Ge atom by a single Pt atom in the second layer of the system. In both cases the images of the two Ge dimers on top of the Pt atom merge into one image, with the location of the overlap on the side where the $\mathrm{Pt}$ atom is located.

For a $\mathrm{Pt}$ atom located at the down side this fusing is only seen for small bias voltages, for large bias the images are almost indistinguishable from the normal Ge dimer images.

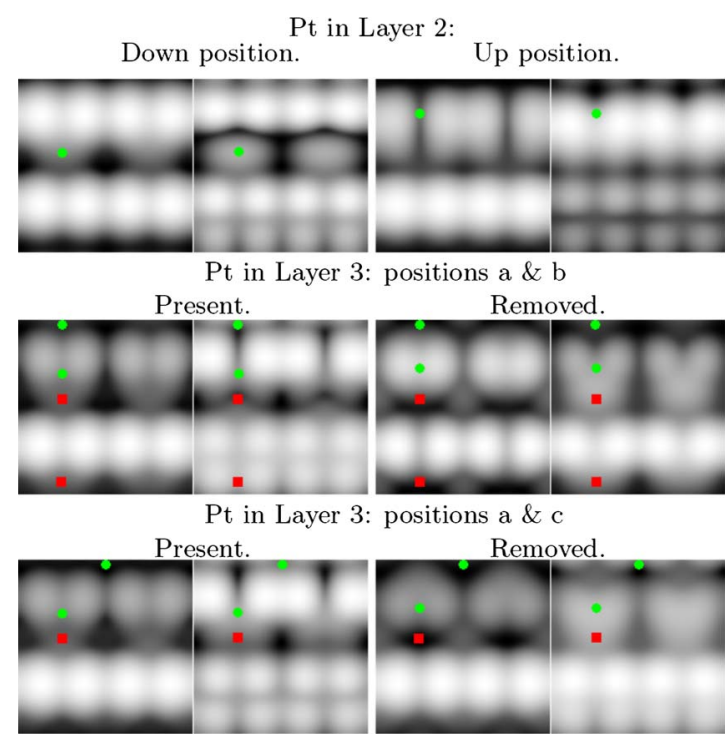

FIG. 13. (Color online) Pseudo-STM images showing filled(left of each pair) and empty- (right of each pair) state images for the geometries containing $\mathrm{Pt}$ in the second and third layer. $\varepsilon$ $=\varepsilon_{F} \pm 0.70 \mathrm{eV}$ is used in these simulations and the maximum $z$ is chosen at $4 \AA$ above the highest atom. The positions of the $\mathrm{Pt}$ atoms are indicated by the (green) disks and the (red) squares indicate the positions of second layer Ge atoms which are pushed up to the first layer level. "Present" and "removed" refers to these Ge atoms being present or having been removed (* configurations of Table III). The bottom dimer row in each picture shows Ge dimers which are not (or barely) influenced by the Pt atoms present. The dimers at the top of each picture show a clear modification caused by the Pt atoms present underneath.

Also, the brightness of the dimers is roughly the same as for the $\mathrm{Ge}$ dimers on a normal $\mathrm{Ge}(001)$ surface.

In case the Pt atom is located at the up side of the dimer row, the position where the two images of the Ge dimers merge moves from being on top of the $\mathrm{Pt}$ atom, for large negative biases, to being on top of the Ge atom in between two Pt atoms, for small negative biases and all positive biases. Also, in this case there is a clear difference in brightness between the normal Ge dimers and the dimers on top of a Pt atom. For negative biases, the normal Ge dimers are most bright while for positive biases, the modified dimers are brighter. The brightest part of the modified dimers extends over the entire dimer for small biases, while for large positive (and negative) bias the brightest part of the image is at the side of the up-Ge atoms. This gives the impression of a dimer located at one side of the dimer row and directed along this dimer row. This is only an impression because these structures are formed by the bending, toward and away from the $\mathrm{Pt}$ atom, of the Ge $p$ orbitals. When all Ge atoms at the up side in the second layer under a dimer row are substituted by $\mathrm{Pt}$ atoms, the images of the above Ge dimers do not merge anymore. Their image only lengthens and becomes a long oval shape which is dimmer than the normal Ge dimer at negative bias and brighter than the normal $\mathrm{Ge}$ dimer at positive bias. This elongated shape persists at positive bias such that there is a clear difference between this image and the image of a normal Ge dimer, for which the single dimer 
image is replaced by two spots, one for each Ge atom.

\section{Pt in the third layer}

Substitution in the third layer can also be done at two inequivalent sites. The first is under the dimer row and the second is in the trough between dimer rows. Table III shows a clear preference for $\mathrm{Pt}$ to be positioned under a dimer row, while a $\mathrm{Pt}$ atom in the trough position is less favorable than the original $b(2 \times 1)$ reconstruction. This again shows the preference of $\mathrm{Pt}$ to be surrounded by Ge atoms. This is understandable since the Pt-Ge bond is energetically favorable over a Pt-Pt or a Ge-Ge bond. Table III also shows that the introduction of a second $\mathrm{Pt}$ atom in the third layer about halves the formation energy per Pt atom, if the second $\mathrm{Pt}$ is located at a next nearest neighbor position. Though this might be expected for a combination of one row and one trough positioned $\mathrm{Pt}$ atom, it is also the case for two $\mathrm{Pt}$ atoms in row positions. Unlike Pt in the second layer, Pt in the third layer prefers to be positioned far from other Pt atoms. Positions $a$ and $e$ seem to be separated enough to remove this negative correlation. Comparing Tables I and III shows clearly that Pt under a dimer row is energetically the most favorable configuration, with formation energy about one order of magnitude larger than the other configurations, in agreement with previous calculations. ${ }^{5}$

Indeed by comparing Tables I and III it can be observed that thermodynamically $\mathrm{Pt}$ atoms prefer to substitute Ge atoms in the third layer. However, this substitution involves breaking the local, bulk-like Ge structure, whereas substitution of Ge by $\mathrm{Pt}$ in the top surface layer involves breaking Ge-Ge surface bonds. From experiments on the Ge(001) surface it is known that at the anneal temperature of $\sim 1050 \mathrm{~K}$, the surface Ge dimers break up. ${ }^{32}$ At this temperature the bulk is still intact, as melting starts at a much higher temperature. This should favor the incorporation of $\mathrm{Pt}$ in the top surface layer. Indeed, experiments show that the formation of the betaterrace is connected to the anneal temperature of $\sim 1050 \mathrm{~K}^{.5,28}$

Geometrically the most impressive change is caused by a $\mathrm{Pt}$ atom under a dimer row at site a (or an equivalent site). The atom pushes the second layer Ge atom (in case of site a, indicated with $k_{1}$ in Fig. 12) at the down side of the row up to the first layer deforming the surface geometry. Also in the case where the geometry contains two $\mathrm{Pt}$ atoms, at positions $a$ and $b$, there is an additional lifting of Ge atom $k_{2}$, which might be considered caused by the $\mathrm{Pt}$ atom at position $a$. The Pt atom (site $a$ ) pushes Ge atom $k_{1}$ up to a first layer position, but it also pushes the Ge atom (site $B$ ) in between the two Pt atoms a little bit away. This Ge atom in turn pushes against the second $\mathrm{Pt}$ atom (site $b$ ) which then lifts Ge atom $k_{2}$ creating a chain effect in which a horizontal displacement is transformed into a vertical one. Table III shows the removal of these Ge atoms (after relaxation to a new equilibrium geometry) costs about 0.6 to $1.1 \mathrm{eV}$ per Ge atom removed. So if the Ge atoms are to be considered as ejected atoms which move away to a position at a step edge, then the energy gained from placing a Pt atom in a row position is almost entirely lost and we again end up in the energy range of the $\beta$ and $\gamma$ geometries. The exception being the geometry placing $\mathrm{Pt}$ atoms in positions $a$ and $e$, which resembles the $\beta_{6}$ geometry, and thus could be considered a possible starting position for the $\beta_{6 u}$ geometry.

The influence on the pseudo-STM pictures is similar as seen for the Pt atoms in the second layer. The charge lobes of the up-Ge dimer atoms are pulled toward one another over the $\mathrm{Pt}$ atom at negative bias while for positive bias they are pushed away. For the down-Ge dimer atoms, the charge lobes are always pulled toward one another over the Pt atom. The merging of the dimer images in the pseudo-STM pictures of Fig. 13 show this nicely. A more important effect caused by this deformation of the charges is the dimming at negative and the brightening at positive biases of the nearby Ge dimers, which can be substantial, as shown in Fig. 13. This indicates a charge transfer from Ge to Pt atoms. Removal of the $k_{\mathrm{x}} \mathrm{Ge}$ atoms forces the down-Ge atoms to make a new bond, which generally is with a third layer atom, either the $\mathrm{Pt}$ atom under the dimer row or the Ge atom in the trough. This removal causes a lowering of the dimer structure into the surface consequently causing a lowering of the electronic structure and hence dimming the dimer image in the pseudo-STM pictures even further. The change in the $z$ position of the dimer atoms is not the only geometrical effect caused by this removal. The dimers also bend toward one another over the location of the removed Ge atom. Consequently the two dimer images in the pseudo-STM picture merge into one single heart-shaped feature, of which the orientation depends on the bias, as can be seen in Fig. 13.

\section{Missing dimers or hidden dimers?}

In the previous two paragraphs it was shown that the introduction of Pt in the second or third layer causes significant changes in the electronic structure of the surface. The difference in height/brightness of the dimer images in the pseudoSTM pictures strongly depends on the simulated bias. Combining the visual information from the STM image with the height differences of the maxima at different biases (both positive and negative) should make it possible to clearly distinguish the different geometries containing Pt in the second and third layer in experiment.

We generated pseudo-STM images for all the structures containing $\mathrm{Pt}$ in the second or third layer, using simulated biases of $-1.50,-0.70,+0.70$, and $+1.50 \mathrm{~V}$ to study the influence of varying bias. For each bias, a set of STM images was generated using different values for $z$ (2.5, 3.0, and $4.0 \AA$ ) to construct surfaces of different constant current, allowing us to study the effect of varying current. In real STM experiments, there are other variables that influence the final STM image such as the tip geometry. As a consequence, one should not interpret the values given below as absolutes, but instead as relative values indicating the trends which should be observed in experiment for these structures.

For a $\mathrm{Pt}$ atom in the second layer at the down location, the maxima calculated for dimers on top of the $\mathrm{Pt}$ atom and dimers without Pt nearby, are roughly the same (less than $0.1 \AA$ ). Here the image at positive bias would give the telltale signature with the dimmed oval structure over a pair of down-Ge dimer atoms, which is $\sim 0.45 \AA$ lower at $+0.70 \mathrm{~V}$ and $\sim 0.3 \AA$ lower than the maxima at $+1.50 \mathrm{~V}$ bias. 
For second layer $\mathrm{Pt}$ atoms at the up location there is the merging of two dimer images into one single rectangular feature, which at low negative bias will shift one full dimer position with regard to the positive bias position. Furthermore, at increasing positive bias the maximum will seem to move to one side of the dimer row. At negative bias, the maximum of the structure will be $\sim 0.25 \AA$ lower than that of a normal Ge dimer, with this height difference becoming larger at smaller bias. At positive bias on the other hand the maximum will be about $0.3 \AA$ higher than that of a normal Ge dimer.

$\mathrm{Pt}$ in the third layer also modifies the electronic structure in a recognizable way. Pt under the dimer row lowers the peaks of the dimer row above by 0.5 to $0.7 \AA$ at negative bias, while a $\mathrm{Pt}$ atom in the trough modifies the Ge dimer, with its up-Ge atom nearby, lowering the maxima of these dimers by only $\sim 0.25 \AA$. For the latter, these dimers are also slightly more asymmetric than the normal ones and at small positive bias the dimer image is still one feature while for a normal dimer the image splits up into two separate atom images. This splitting still occurs for the modified dimer but at a larger positive bias. At positive bias the modified dimers are highest for both Pt positions. For low positive biases they are $\sim 0.25 \AA$ higher, than the normal dimers, while for large positive bias this difference shrinks to only $\sim 0.1 \AA$. Removal of the Ge atoms, which were pushed to the top layer, changes the electronic structure dramatically from the normal Ge dimer image. In each case two dimer images are merged into one single large heart-shaped feature which is about 0.6 to $0.7 \AA$ lower than the maxima of the normal dimers at negative bias, and about $0.8 \AA$ lower at small positive bias. At large positive bias this difference reduces to $\sim 0.25 \AA$ at which point the maximum of the structure is located at one side of the dimer row, giving the feature a somewhat butterflylike appearance.

Combinations as seen in Fig. 13 give similar results. For example, the height difference of the dimer images, for Pt in the third layer at positions $a$ and $c$ in the filled-state image is $\sim 0.8 \AA$ and in the empty-state image this is $\sim 0.2 \AA$. When the $k_{1}$ Ge atom is removed, the filled-state image now gives a height difference of $\sim 1.0 \AA$ while the empty-state image shows one of $\sim 0.6 \AA$. For both filled- and empty-state images the normal dimers are highest.

Although we used a very simple model to calculate the pseudo-STM pictures, the values obtained suggest that some of these geometries could cause experimentally observed features in the $\alpha$ terrace such as indented dimers or two dimer vacancies. Indented dimers are a structure two dimers long and based on the above results we conclude that this feature is caused by a Pt atom in a second layer up position or a third layer trough position. Two dimer vacancies on the other hand might not be actual missing dimers but merely due to the charge transfer from Ge-Ge dimers to a Pt atom in a third layer row position underneath. At this moment the above remains uncertain. A high resolution STM study over varying bias of these defects however, should make a conclusive statement on this subject possible.

\section{CONCLUSIONS}

We study the possible geometries containing 0.25 and 0.5 ML of Pt substituted in the top layer of a $\mathrm{Ge}(001)$ reconstructed surface. Zero-temperature $a b$ initio calculations show that the formation of Pt-Ge dimers constitutes the most favorable reconstructions. They also show the spontaneous formation of alternate buckling in most geometries due to the introduction of Pt. Comparison of calculated STM images with experimental observations is necessary to show the $\beta$ terrace has a $\beta_{6 u}$ geometry, which has some similarities with the $\mathrm{Ge}(001) c(4 \times 2)$ reconstruction. It is shown that the Pt-Ge dimers are tilted in antiphase to the Ge-Ge dimers, and a thorough description the $\beta_{6 u}$ geometry is given in terms of twisting, due to the antiphase buckling of dimers in a dimer row, bending, due to the antiphase buckling of dimers in adjacent dimer rows, and shift, due to the asymmetry of having two types of dimers present on the surface. Additionally, we study geometries containing $\mathrm{Pt}$ atoms substituted in the second and third layer and show the most stable configuration to be one with Pt atoms located under the Ge dimer row. It is also shown that $\mathrm{Pt}$ atoms in this position push a second layer $\mathrm{Ge}$ atom to the surface and that the removal of this atom does not increase the formation energy but instead decreases it. In all these "other" geometries, with exception of those where $\mathrm{Ge}$ atoms were removed, the presence of $\mathrm{Pt}$ atoms both modifies and dims the Ge dimers on top for negative bias and brightens them for positive bias. The height differences we find indicate that these other geometries could be responsible for experimentally observed indented dimers and two dimer vacancies. Also, the calculated STM images of these other geometries show clearly that they cannot be responsible for the $\beta$ terrace, though some are energetically more favorable, indicating that the $\beta_{6 u}$ geometry is a local minimum.

\section{ACKNOWLEDGMENTS}

We acknowledge Harold Zandvliet and Arie van Houselt for many useful discussions, and for making available their experimental data. This work is part of the research program of the "Stichting voor Fundamenteel Onderzoek der Materie" (FOM) and the use of supercomputer facilities was sponsored by the "Stichting Nationale Computer Faciliteiten" $(\mathrm{NCF})$, both financially supported by the "Nederlandse Organisatie voor Wetenschappelijk Onderzoek" (NWO). 
${ }^{1}$ J. V. Barth, G. Constantini, and K. Kern, Nature (London) 437, 671 (2005) and references therein.

${ }^{2}$ X. F. Lin, K. J. Wan, J. C. Glueckstein, and J. Nogami, Phys. Rev. B 47, 3671 (1993).

${ }^{3}$ M. Kageshima, Y. Torii, Y. Tano, O. Takeuchi, and A. Kawazu, Surf. Sci. 472, 51 (2001).

${ }^{4}$ T. Shimakura, H. Minoda, Y. Tanishiro, and K. Yagi, Surf. Sci. 407, L657 (1998).

${ }^{5}$ O. Gürlü, O. A. O. Adam, H. J. W. Zandvliet, and B. Poelsema, Appl. Phys. Lett. 83, 4610 (2003).

${ }^{6}$ N. Öncel, A. van Houselt, J. Huijben, A. S. Hallback, O. Gürlü, H. J. W. Zandvliet, and B. Poelsema, Phys. Rev. Lett. 95, 116801 (2005).

${ }^{7}$ J. Schäfer, D. Schrupp, M. Preisinger, and R. Claessen, Phys. Rev. B 74, 041404(R) (2006).

${ }^{8}$ R. H. M. Smit, C. Untiedt, A. I. Yanson, and J. M. van Ruitenbeek, Phys. Rev. Lett. 87, 266102 (2001).

${ }^{9}$ J. Wang, M. Li, and E. I. Altman, Phys. Rev. B 70, 233312 (2004).

${ }^{10}$ J. Wang, M. Li, and E. I. Altman, Surf. Sci. 596, 126 (2005).

${ }^{11}$ D. E. P. Vanpoucke and G. Brocks, Phys. Rev. B 77, 241308(R) (2008).

${ }^{12}$ A. A. Stekolnikov, F. Bechstedt, M. Wisniewski, J. Schafer, and R. Claessen, Phys. Rev. Lett. 100, 196101 (2008).

${ }^{13}$ P. E. Blöchl, Phys. Rev. B 50, 17953 (1994).

${ }^{14}$ G. Kresse and D. Joubert, Phys. Rev. B 59, 1758 (1999).

${ }^{15}$ D. M. Ceperley and B. J. Alder, Phys. Rev. Lett. 45, 566 (1980).

${ }^{16}$ G. Kresse and J. Hafner, Phys. Rev. B 47, 558 (1993).

${ }^{17}$ G. Kresse and J. Furthmüller, Phys. Rev. B 54, 11169 (1996).

${ }^{18}$ H. J. Monkhorst and J. D. Pack, Phys. Rev. B 13, 5188 (1976).

${ }^{19}$ M. Needels, M. C. Payne, and J. D. Joannopoulos, Phys. Rev. Lett. 58, 1765 (1987).

${ }^{20}$ M. Needels, M. C. Payne, and J. D. Joannopoulos, Phys. Rev. B 38, 5543 (1988).

${ }^{21}$ S. J. Jenkins and G. P. Srivastava, J. Phys.: Condens. Matter 8, 6641 (1996)

${ }^{22}$ S. C. A. Gay and G. P. Srivastava, Phys. Rev. B 60, 1488 (1999).

${ }^{23}$ For the sake of simplicity and ease of writing we refer to any bound atom pair on the surface and in its top layer as a dimer, even if it consists of two different atoms. If the atom types are important we will refer to the dimer as an $X-Y$ dimer, with $X$ and $Y$ its constituent atoms. Also a shorter notation will be used where the term "Ge dimers" is used to refer to Ge-Ge dimers and Pt the term "Pt dimers" is used to refer to dimers containing at least $1 \mathrm{Pt}$ atom. For the latter it will follow from the context if this refers to Pt-Ge dimers or Pt-Pt dimers.

${ }^{24}$ Note that the $u$ and $d$ index only indicate the position in the $\mathrm{Ge}(001)$ reconstruction geometry and tells nothing about the relative $z$ position of the $\mathrm{Pt}$ atoms with regard to the $\mathrm{Ge}$ atom in the specific Pt-Ge dimer.

${ }^{25}$ An alternative way of calculating the formation energy (which gives exactly the same results) is by calculating the surface formation energy $E_{s b}$ of the $\beta$ surface using the expression,

$$
E_{s b}=E_{T}\left[\beta_{\mathrm{x}}\right]-N_{\mathrm{Pt}} E_{\mathrm{Pt}}-N_{\mathrm{Ge}} E_{\mathrm{Ge}},
$$

where $E_{T}\left[\beta_{\mathrm{X}}\right]$ is the total energy of the $\beta_{\mathrm{x}}$ surface, $N_{X}$ the number of atoms of type $X$ in the slab with $E_{X}$ the corresponding bulk energy per atom. This formation energy we can now consider to be the sum of the formation energies of the separate surface dimers. Because the surface consists of two types of dimers, it is reasonable to assume that they will contribute differently to the total formation energy. The formation energy of a $\mathrm{Ge}-\mathrm{Ge}$ dimer is found by calculating the formation energy per surface dimer for a $\mathrm{Ge}(001) b(2 \times 1)$ surface reconstruction. Substraction of the formation energy caused by the Ge-Ge dimers gives

$$
E_{s b 2}=E_{s b}-N_{\mathrm{Ge} \text {-dimers }} E_{s \mathrm{Ge} \text {-dimer }},
$$

with $N_{\text {Ge-dimers }}$ the number of Ge-Ge dimers in the $\beta$ surface and $E_{s \mathrm{Ge} \text {-dimer }}$ the formation energy of a Ge dimer in the $b(2 \times 1)$ surface reconstruction. The resulting energy $E_{s b 2}$ can then be considered the contribution by the Pt-Ge dimers to $E_{s b}$. If we again subtract the formation energy $E_{s \mathrm{Ge} \text {-dimer }}$ from the calculated energy contribution pet Pt-Ge dimer, $E_{s b 2} / N_{\text {Pt-dimers }}$, the formation energy per Pt-Ge dimer as given in Eq. (1) is obtained. In this derivation the physical meaning of the formation energy becomes clear: "the energy gained/needed to replace a Ge-Ge dimer by a Pt containing dimer." This is true under the assumption that there is no contribution to the surface energy due to dimer-dimer interactions or (as we will do) if all changes herein are attributed to the Pt containing dimers and they are assumed to be equal in a single geometry. This allows us to estimate the energy contributions to the surface formation energy due to inter and intradimer row interactions, by comparison of similar geometries.

${ }^{26}$ M. K. Niranjan, L. Kleinman, and A. A. Demkov, Phys. Rev. B 75, 085326 (2007).

${ }^{27}$ J. Tersoff and D. R. Hamann, Phys. Rev. B 31, 805 (1985).

${ }^{28}$ O. Gürlü, Ph. D. thesis, University of Twente (2004).

${ }^{29}$ A. van Houselt, N. Öncel, B. Poelsema, and H. Zandvliet, Nano Lett. 6, 1439 (2006).

${ }^{30}$ The zero, or nearly zero, band gap for germanium is a known failure of LDA. Although a band gap can be opened using for example the GW method, it is not necessary for the qualitative comparison we are making here.

${ }^{31}$ O. Gürlü, H. J. W. Zandvliet, B. Poelsema, S. Dag, and S. Ciraci, Phys. Rev. B 70, 085312 (2004).

${ }^{32}$ X. Zeng and H. E. Elsayed-Ali, Surf. Sci. 497, 373 (2002). 